Proceedings of the 17th Czech and Slovak Conference on Magnetism, Košice, Slovakia, June 3-7, 2019

\title{
Manipulation of Domain Wall Shape in Thin Magnetic Wire by Current Annealing
}

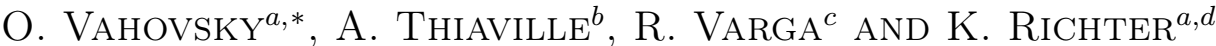 \\ ${ }^{a}$ Institute of Physics, Faculty of Sciences, P.J. Safarik University, Park Angelinum 9, 04154 Kosice, Slovakia \\ ${ }^{b}$ Laboratoire de Physique des Solides, Universite Paris-Sud, Universite Paris-Saclay, \\ CNRS, UMR 8502, F-91405 Orsay Cedex, France \\ ${ }^{c}$ CPM-TIP, P.J. Safarik University, Park Angelinum 9, 04154 Kosice, Slovakia \\ ${ }^{d}$ Institute for Materials Science, University of Kiel, Kaiserstrasse 2, 24143 Kiel, Germany
}

\begin{abstract}
Here, we present magneto-optical studies of a surface domain wall (DW) shape in as-cast and heat-treated glass-coated microwires (MW). Technique optimized for the study of thin magnetic cylinders is used for local mapping of the magnetization process on wire's circumference. Consequently, the shape of the surface DW can be visualized at an arbitrary point along the wire's length. Samples undergo sequential heat treatment, and DW shape in the same sample before and after heat treatment is compared. Variation in DW tilting angle and length is observed as a result of annealing.
\end{abstract}

DOI: 10.12693/APhysPolA.137.821

PACS/topics: domain wall, microwire, annealing

\section{Introduction}

Spin dynamics in micro and nanoscale is in centre of interest not only due to its applicability [1], but also from a fundamental point of view [2]. Most of the recent work is focused on understanding and tailoring the DW dynamics [3] by variation of the intrinsic properties of MW. Particularly, magnetoelastic anisotropy is modified by various heat treatments, releasing the stresses induced into the material in the manufacturing process.

Generally, the heat treatment of Fe-rich MW leads to the initial abrupt increase of the mobility DW. Nevertheless, further annealing of the sample brings rather slight increase of DW [3], as well as the decrease in coercivity [4]. This has been explained by the relaxation of the internal mechanical stresses, which decreases the magnetoelastic anisotropy and DW energy.

The knowledge of the DW shape is critical for understanding the DW dynamics enhancement by annealing. Usually, assumptions about the DW shape are based on Sixtus-Tonks experiments $[5,6]$, or direct observations by magneto-optic Kerr effect (MOKE) microscopy [7]. The disadvantage of the Sixtus-Tonks-like experiments lies in the fact that obtained information is averaged over the whole MW volume. Thus, fine local features of the DW cannot be resolved. On the other hand, observations of curved surfaces by MOKE microscopy are rather challenging since the field of view being naturally limited by the numerical aperture of the objective. Hence, only a small part of the surface can be observed with the use of the microscope.

\footnotetext{
* corresponding author; e-mail: oliver.vahovsky@student.upjs.sk
}

In this paper, we study the effect of thermal treatment on a surface DW tilting angle in glass-coated MW. Timeresolved measurements of the propagating surface DW are carried out on the same place of the sample, before and after gradual current annealing. MOKE data are analysed, and processed to visualize a shape of a propagating surface DW, including local changes in magnetic reversal along the wire's circumference, as well as its length.

\section{Experimental}

Glass-coated $\mathrm{Fe}_{77.5} \mathrm{Si}_{7.5} \mathrm{~B}_{15}$ MWs fabricated by TaylorUlitovsky method are investigated. The length of the samples is $l=3 \mathrm{~cm}$, the metallic core diameter is $d=17 \mu \mathrm{m}$, and the total diameter is $D=57 \mu \mathrm{m}$.

Our observations of a surface DW shape have been carried out by an experimental setup introduced in [8]. The sample is placed vertically on the axis of two identical coils separated by the distance $d=15 \mathrm{~mm}$, and a part of its surface is illuminated by a LASER beam. MOKE intensities from two different spots on the sample's circumference are sensed by photodetectors. The coil system including the sample is rotatable around its axis. The DW is trapped in a magnetic potential well, created in such way that one of the coils is supplied by the DC current, while the other by a triangular AC current with the DC offset. The MOKE configuration used is sensitive to the longitudinal component of surface magnetization [8], which is controlled by the magnetization direction of the inner core just below the observed area [9].

The heat treatment consists of DC current annealing for a period of $t=15 \mathrm{~min}$, followed by a reduction of the magnitude of current at the rate of $1 \mathrm{~mA} / 30 \mathrm{~s}$ to enable moderate cooling of the MW. The whole procedure 


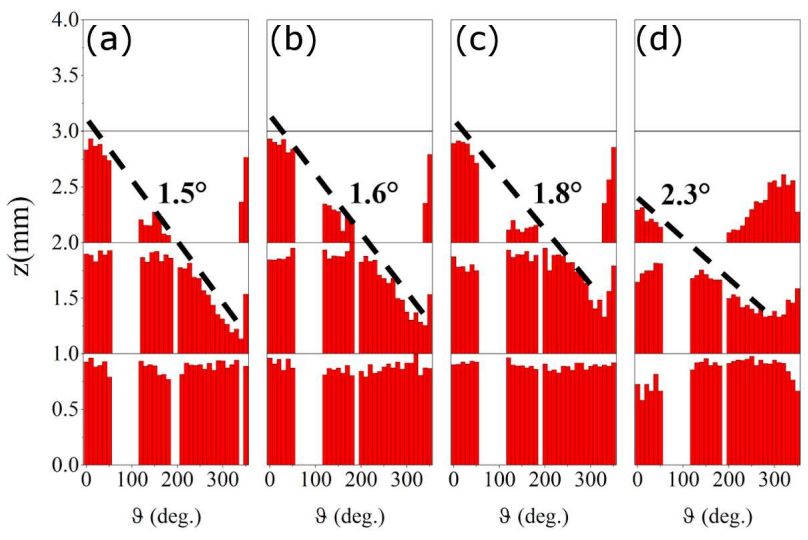

Fig. 1. (a) Surface DW shape in each of the four vertically adjacent points $(0 \mathrm{~mm}<z<4 \mathrm{~mm})$ in the same external magnetic field (as-cast state). $\vartheta$ denotes the position on the MW circumference, while $z$ is the coordinate along the wire's length. The dashed line serves as a guide to the eye to illustrate the position of the DW, while the approximate angle of inclination found by linear fit is denoted (b) annealed at $I_{a}=20 \mathrm{~mA}$ for $15 \mathrm{~min}$, (c) annealed at $I_{a}=40 \mathrm{~mA}$ for $15 \mathrm{~min}$, and (d) annealed at $I_{a}=60 \mathrm{~mA} \mathrm{~mA}$ for $15 \mathrm{~min}$.

is repeated, thus the magnitude of the annealing current in each iteration increases. The measurement of the DW shape is carried out between the annealing steps at the same place on the wire.

As stated in [8], to reveal the shape of the propagating surface DW, one should rotate the MW around its axis in small steps $\left(\alpha=10^{\circ}\right)$. Contrary to our previous measurements, here we do not confine ourselves to a wire rotation, but we also perform scanning in several adjacent vertical positions, before and after annealing (Fig. 1a-d).

\section{Results and discussion}

Considerable improvement of dynamic properties of Fe-rich amorphous microwires as a result of annealing $[3,4]$ motivated us to study the effect of heat treatment on the structure of a domain wall. The DW propagation in thin magnetic wires is described by a phenomenological model of DW motion, where the DW velocity is a linear function of the applied magnetic field $H$ [10]. This is expressed by

$$
v=S\left(H-H_{0}\right) \quad \text { with } \quad S=\frac{2 \mu_{0} M_{S}}{\beta},
$$

where $H_{0}$ is the critical field, $S$ is so-called the DW mobility, $\mu_{0}$ is the permeability of vacuum, $M_{s}$ is the saturation magnetization, and $\beta$ is the DW damping coefficient. In the case of thin magnetic wires, $\beta$ can be expressed as: $\beta=\frac{\alpha}{\gamma \Delta}$, where $\gamma$ is the gyromagnetic ratio, $\alpha$ is the Gilbert damping parameter, while $\Delta$ denotes the DW width. The estimated DW width is $\Delta \approx \sqrt{A / K}$ with the exchange constant $A$, and the uniaxial anisotropy constant $K$. Hence, DW mobility is inversely proportional to the damping coefficient, and thus to the square root of uniaxial anisotropy. A decrease of $K$ due to annealing could result in the variation of the width of the domain wall through a relation between $K$ and $\Delta$. Potentially, the change in the shape and structure of the domain wall may also occur.

DW shape measurements are summarised in Fig. 1, which is formed of 16 plots, each representing the unrolled surface of the MW in either different longitudinal position (vertical dimension), or different step in gradual thermal treatment (horizontal dimension). Each plot in Fig. 1 refers to separate measurement, and is a combination of normalized MOKE intensities obtained from sensing spots along the wire's circumference in the same external magnetic field. White spaces (e.g., for $50^{\circ}<\vartheta<120^{\circ}$ ) correspond to blind angles resulting from the design of the experimental setup (i.e., laser beam reflecting off the sample holder or coil support). Surface magnetization of Fe-rich MWs includes significant longitudinal component [11], which we are able to sense (red vs. blank space in Fig. 1), and radial component $[7,9,12]$. However, the domain structure of the unrolled surface of the microwire does not have to necessarily correspond to a volume domain wall shape, which can be more complex $[5,6]$.

As can be readily observed, the surface DW in the ascast state is considerably tilted $\left(\approx 1.5^{\circ}\right.$ from axial direction). The angle increases by approximately $7 \%$ and $20 \%$ following the first and the second annealing step, respectively (Fig. 1).

Multiple works have shown an increase in DW mobility $S$ as a result of thermal treatment [3, 4], explained by relaxation of the internal stresses and uniaxial anisotropy $K$. As an increase in the DW width $\Delta$ is expected with decreasing $K$, thus the change in the spin structure and shape of the DW is anticipated.

Apart from increase of tilting angle, and hence decrease of the DW length (from $l_{\text {as }- \text { cast }}=2 \mathrm{~mm}$ to $\left.l_{\text {annealed }}(40 \mathrm{~mA})=1.5 \mathrm{~mm}\right)$, there is no qualitative distinction in DW shape in as-cast and annealed sample up to annealing currents of $I=40 \mathrm{~mA}$ (see Fig. 1a-c).

However, the domain structure of the MW changes abruptly after the final annealing step, pointing to a more complicated multidomain state. Data could be explained by partial crystallization of the wire, which was shown to inhibit magnetic bistability [14]. Hence, the magnetization reversal by single DW propagation should be no longer expected. Moreover, circular Oersted field is present during annealing, and some induced circular anisotropy as a result of annealing could be considered [13]. Our technique, as was already mentioned, is sensitive to neither radial nor a circular component of magnetization, hence the effect of Oersted field was not studied in more detail.

\section{Conclusions}

Here, we study the effect of thermal treatment on a DW shape in FeSiB MW through MOKE. Despite the fairly large increase in DW mobility in this kind of 
wires, the expected abrupt change in the surface DW has not been observed. A slight change in tilting angle is measured, which was accompanied by the decrease of the lenght of the domain wall.This indicates, that magnetostatic contribution to domain wall shape, less sensitive to annealing, should be considered along with the magnetoelastic contribution. On the other hand, the same effect can be the result of a strong DW interaction with surface defects, thus dominating its final shape and spin structure.

\section{Acknowledgments}

This work was supported by the bilateral SlovakFrench project APVV SK-FR-2017-024, APVV-16-0079, APVV-17-0184, VEGA 1/0195/18, VEGA 1/0053/19, VVGS-PF-2018-798 and VVGS-PF-2019-1046.

\section{References}

[1] V. Lopez-Dominguez, M.A. Garcia, P. Marin, A. Hernando, Sci. Rep. 7, 9394 (2017).

[2] K. Richter, A. Krone, M.A. Mawass, B. Kruger, M. Weigand, H. Stoll, G. Schutz, M. Klaui, Phys. Rev. B 94, 2 (2016).

[3] A. Zhukov, J.M. Blanco, M. Ipatov, A. Talaat, V. Zhukova, J. Alloys Compd. 707, (2017).
[4] J. Onufer, J. Ziman, P. Duranka, M. Kladivova, Physica B. 540, (2018).

[5] L.V. Panina, M. Ipatov, V. Zhukova, A. Zhukov, Physica B 407, 9 (2012).

[6] F. Beck, J.N. Rigue, M. Carara, J. Magn. Magn. Mater. 435, (2017).

[7] A. Chizhik, A. Stupakiewicz, A. Zhukov, J. Gonzalez, Phys. Status Solidi A 213, 2 (2016).

[8] O. Vahovsky, R. Varga, K. Richter, J. Magn. Magn. Mater. 483, (2019).

[9] N.N. Orlova, V.S. Gornakov, A.S. Aronin, J. Appl. Phys. 121, 205108 (2017).

[10] N.L. Schryer, L.R. Walker, J. Appl. Phys. 45, 5406 (1974).

[11] K. Richter, A. Thiaville, R. Varga, Phys. Rev. B 96. 064421 (2017).

[12] O.I. Aksenov, G.E. Abrosimova, A.S. Aronin, N.N. Orlova, M.N. Churyukanova, V.A. Zhukova, A.P. Zhukov, J. Appl. Phys. 122, 235103 (2017).

[13] V. Zhukova, J. M. Blanco, M. Ipatov, J. Gonzalez, M. Churyukanova, A. Zhukov, Scr. Mater. 142, 10 (2018).

[14] A. Zhukov, A.F. Cobeno, J. Gonzalez, A. Torcunov, E. Pina, M.J. Prieto, J.M. Blanco, V. Larin, S. Baranov, J. Magn. Magn. Mater. 203, 238 (1999). 